\title{
CFD modelling and PIV experimental validation of flow fields in urban environments
}

\author{
Renata Gnatowska ${ }^{1,}$, Marcin Sosnowski ${ }^{2}$, and Václav Uruba ${ }^{3}$ \\ ${ }^{1}$ Czestochowa University of Technology, Faculty of Mechanical Engineering and Computer Science, \\ 21 Armii Krajowej Av, 42-200 Czestochowa, Poland \\ ${ }^{2}$ Jan Dlugosz University in Czestochowa, Faculty of Mathematics and Natural Sciences, 13/15 Armii \\ Krajowej Av, 42-200 Czestochowa, Poland \\ ${ }^{3}$ Academy of Sciences of the Czech Republic, Institute of Thermomechanics, v.v.i.; Dolejškova \\ 1402/5, 18200 Praha 8, Czech Republic \\ University of West Bohemia, Department of Power System Engineering, Univerzitní 22, 30614 Plzeň \\ Czech Republic
}

\begin{abstract}
The problem of flow field in the urban boundary-layer (UBL) in aspects of wind comfort around buildings and pollutant dispersion has grown in importance since human activity has become so intense that it started to have considerable impact on environment. The issue of wind comfort in urban areas is the result of complex interactions of many flow phenomena and for a long time it arouses a great interest of the research centres. The aim of article is to study urban atmospheric flow at the local scale, which allows for both a detailed reproduction of the flow phenomena and the development of wind comfort criteria. The proposed methodology involves the use of PIV wind tunnel experiments as well as numerical simulations (Computational Fluid Dynamics, CFD) in order to enhance understanding of the flow phenomena at this particular scale in urban environments. The analysis has been performed for the $3 \mathrm{D}$ case of two surface-mounted buildings arranged in tandem, which were placed with one face normal to the oncoming flow. The local characteristics of flow were obtained by the use of commercial CFD code (ANSYS Fluent). The validation was carried out with reference to the PIV results.
\end{abstract}

\section{Introduction}

Increased interest in the problem of flow fields in urban environments has been observed over the past few decades. It is governed by potential applications of the results in the design process of new buildings as well as optimization of the existing urban areas and city centres. Nowadays, this issue plays an important role in environmental protection, it concerns in particular the so called wind comfort around and between buildings, the life quality in urban areas as well as specific economic aspects of utilization of the defined zones. The studies of the wind environment around buildings are generally performed as wind tunnel experiments since it is the most well-established way to simulate a natural

\footnotetext{
* Corresponding author: gnatowska@imc.pcz.czest.pl
} 
wind. In the past decades, numerous studies showed that the prediction of flow field in the surroundings of high-rise buildings in urban environments is very effective by the use of Computational Fluid Dynamics (CFD) [1-4]. Most of the studies have dealt with complicated, real configurations so it is uncertain whether the prediction of CFD is accurate. However, one of the most important aspects of this type of simulations is that the methods and results need to be validated before such predictions can be relied on. Therefore, PIV verification and validation of CFD studies are imperative, as well as detailed sensitivity studies that can provide guidance in the selection of computational parameters for future CFD analysis [5].

There is relatively little verification of CFD which simulate the air flows around objects in different configurations. For a real situation, the accuracy of CFD cannot be tested because of the lack of related experimental data.

CFD simulation of wind flow around 3D buildings started with fundamental studies for isolated buildings, often with a cubical shape [6-8]. Other often investigated configurations are cubic obstacles in a tandem arrangement, where the flow field is further complicated by mutual interference [8-10]. The flow structure around surface mounted obstacles depends on various factors: the grid resolution, the influence of the boundary conditions, the oncoming boundary layer thickness $\delta$ related to the height of obstacles $H$ and by comparing the performance of various types of turbulence models in steady RANS simulations [7-8]. Other important factors are also the oncoming flow turbulence, the non-dimensional distance ratio $(S / H)$ and the height ratio of the two consecutive bodies. Tall buildings, defined as those which protrude above their neighbours, act as scoops to collect the wind over much of their height and deflect it to the ground level [11]. In front of a building exposed to the wind, a vortex forms below the stagnation point. This vortex is shed on each side of the building and it can produce excessively high winds at ground level, causing discomfort or even danger.

The present paper provides a validation study of influence of the geometry of buildings arrangement on flow fields. The CFD simulation results are compared with Particle Image Velocimetry (PIV) wind tunnel measurements. The analysis has been performed for the 3D case of two surface-mounted buildings arranged in tandem, which were placed with one face normal to the oncoming flow. The local characteristics of flow were obtained by the use of commercial CFD code (ANSYS Fluent).

\section{The applied research methods}

The program of the study consists of wind-tunnel experiments and numerical simulation. In the frame of the numerical study, the three-dimensional steady RANS simulations have been carried out using a commercial CFD code, FLUENT v.17, with the realizable version of k-epsilon turbulence model. According to the literature $([3-5,12])$ this model is widely used for analysis of flows for different configurations of buildings.

The geometries of the analysed cases of two rectangular in-line surface-mounted square cylinders immersed in a boundary layer are sketched in figure 1 . The measurements were carried out for configurations of two elements with different height, aligned in one line and the distance between them was $S / D=1.5$. The results presented in this work relate to a fixed ratio of object height $H_{1} / H_{2}=0.6$ and value of their "immersion" in boundary layer $H_{2} / \delta=1$.

\subsection{CFD simulations: settings and parameters}

The dimensions of the computational domain were determined based on the best practice guidelines $([13,14])$. The upstream distance between inlet plane and windward 
building facade is $7.5 \mathrm{D}$, while the downstream distance between leeward building facade and outlet plane is $29 \mathrm{D}$. The distance between the side walls of the building and the side planes of the domain is $4.5 \mathrm{D}$ on both sides. For computation purposes, the flow domain is divided into a number of hexahedral cells. The grid is clustered near the object and the spacing is increased to a growth ratio of 1.2 in the surroundings of the building model. The grid is obtained based on grid-sensitivity analysis. Three grids were made by increasing the resolution by the factor of 2 , from Grid A (182388 cells) thru Grid B (341571 cells) to Grid C (523634 cells). The results obtained on these three grids are compared in terms of the pressure on the surface of first and second object. These tests indicate that the resolution of the finer grid (Grid C) is suitable.

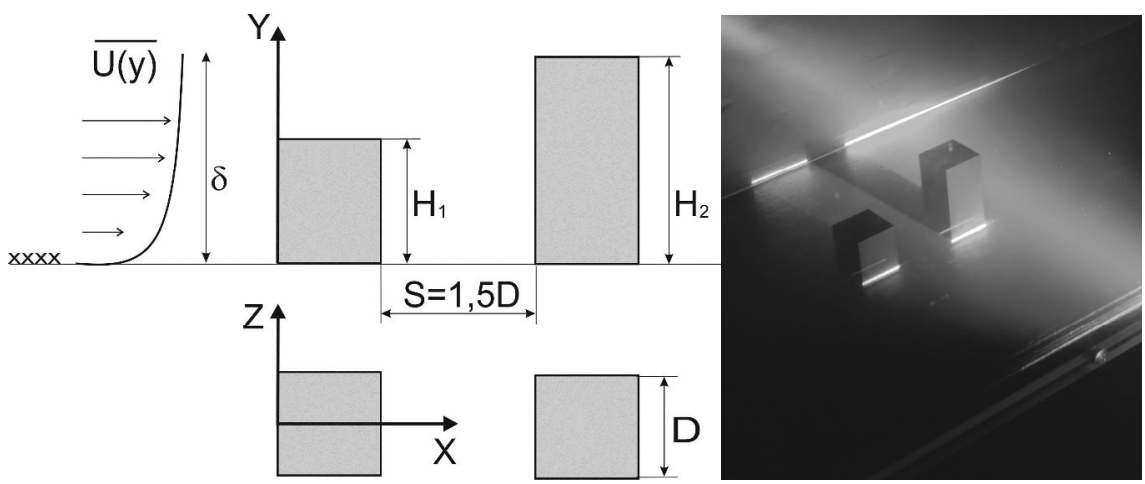

Fig. 1. Scheme of analysed flow around two tandem arrangement objects and the photo of wind tunnel setup.

The inlet mean velocity profile has been approximated after wind tunnel experiment using the power distribution:

$$
U(y)=U_{\delta}(y / \delta)^{\alpha}
$$

where, $\alpha$ characterizes the terrain type ( $\alpha=0.15$; suburban boundary layer). In addition to the inlet velocity profile, the $\mathrm{k}-\varepsilon$ realizable turbulence model requires an appropriate distribution of the turbulent kinetic energy $k$ and the dissipation rate $\varepsilon$ which together define the velocity and length scale of the turbulent motion. One of the best methods to describe the inlet boundary conditions is the method proposed by Richards and Hoxey [15]. The turbulent kinetic energy profile is estimated from the $u_{r m s}$ profile measured in wind tunnel. The turbulence dissipation rate is given by Eq. (2) [14]:

$$
\varepsilon(y)=u *^{3} / \kappa y
$$

where, $\kappa$ is the von Kármán constant equal 0.41 , and $u *$ is the friction velocity $u^{*}=$ $0.04 \mathrm{U}$ [16]. This, method has been applied for the calculations described in this paper.

The standard wall functions in FLUENT is based on the work of Launder and Spalding [17], and have been used in this calculations. The governing equations are solved using the control volume method. Pressure-velocity coupling by SIMPLE algorithm was used as a solution method with second-order pressure interpolation. Second-order discretisation schemes are used for both the convection terms and the viscous terms of the governing equations. In order to assess the convergence of the solution, both the scaled residuals and the streamwise wind speed in selected points of the domain were monitored as a function of the number of iterations, in accordance to the guidelines by Franke et al. [13] and Tominaga et al. [14]. Convergence was obtained when the scaled residuals levelled off and reached a 
minimum of $10^{-6}$ for $\mathrm{x}-, \mathrm{y}-, \mathrm{z}$-velocity, $\mathrm{k}$ and $\varepsilon$ and $10^{-4}$ for continuity. The static pressure on objects surfaces was also monitored.

\subsection{Wind tunnel experiments}

The experiment with Particle Image Velocimetry (PIV) was carried out in an opencircuit wind tunnel at the Institute of Thermomechanics of Academy of Sciences of the Czech Republic. The analysed configuration of buildings model was located within boundary layer generated by the facility output from the contraction nozzle of a crosssection of $250 \times 250 \mathrm{~mm}^{2}$. The test section of wind tunnel is $3 \mathrm{~m}$ long, but the measurements were performed in the central part of tunnel. For seeding particles, a standard SAFEX fog generator has been used. The laser beam from a dual-head Nd:YLF laser with a pulse energy of $10 \mathrm{~mJ}$ at $2 \mathrm{kHz}$ was made into a thin light-sheet by optics to illuminate tracer particles. A Dantec CCD camera (SpeedSense 611) was mounted to capture pairs of particle images at a frame rate of $100 \mathrm{~Hz}$. In order to evaluate the flow around the tandem cubes, two-component particle image velocimetry (PIV) is employed. The PIV measurements provide a velocity vector field with 2 velocity components in horizontal streamwise and spanwise $\mathrm{x}$ - and z-direction $\left(U_{x}\right.$ and $\left.U_{z}\right)$ at different section characterised by $z / D$ parameter with step $z=0.005 \mathrm{~m}$ from the axis system. The farthest measuring section from the axis of building system was located at $z / D=2$. For both numerical and experimental cases the Reynolds number was set to $R e_{D}=9000$ based on the free-stream velocity $U=5.5 \mathrm{~m} / \mathrm{s}$ and the cube width $D=0.025 \mathrm{~m}$. Figure 2 shows the measured mean wind velocity and velocity fluctuations, as well as the fitted numerical distributions, which will be used as inlet boundary conditions in the carried out CFD simulation. The vertical turbulence structure $\left(u_{\mathrm{rms}}\right)$ have not got the ideal shape of "traditional" boundary layer, because it have been measured at the inlet to the test section in wind tunnel at the Institute of Thermomechanics, Academy of Sciences of the Czech Republic.

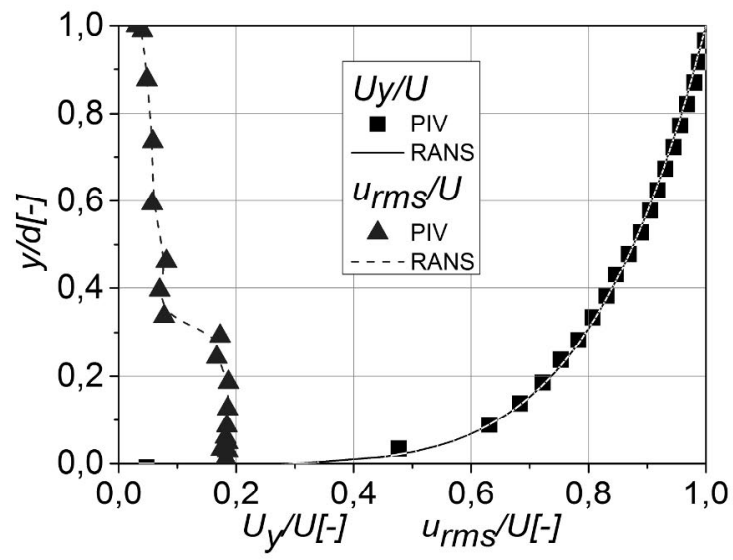

Fig. 2. The inlet profiles of mean wind speed $\left(U y / U\right.$ ) and velocity fluctuations $u_{\mathrm{rms}} / U$ measured in the wind tunnel at the building position (PIV) and the inlet profiles used in the CFD simulations (RANS).

\section{CFD results and validation with wind tunnel measurements}

For multiple bluff bodies arrays, the flow field is more complex than the flow around a single object due to mutual interactions and interference phenomena. The flow around the three-dimensional bluff-body is composed of a range of separation and adherence points classified as singular nodal and saddle points. One can distinguish here a horseshoe vortex 
and post-edge vorties located a close distance from the rear side of the object. The flow changes to strongly complicated if another object is placed in the aerodynamic wake, because of both the periodicity of vortex shedding and the cyclic oscillatory air flow between the obstacles. The results from the CFD simulation are compared with the experimental PIV data in terms of wind velocity ratio, velocity-vector fields and streamlines around two surface-mounted buildings arranged in tandem along the vertical planes.

The CFD simulations that are shown in the validation study are the ones considered the most accurate. These are the results obtained with Grid C (see section 2). Note that the experimental velocity-vector fields in the immediate vicinity of the ground are considered less reliable due to effects of shadows or reflections that might have produced uncertainties in the PIV measurements at these positions. Also the very high wind speed gradients in this area might have caused additional uncertainties in the PIV results.

The CFD validation results are shown in Figs. 3 and 4. These figures show the flow through selected sections in transverse direction of flow in analysed tandem configuration. The flow field is symmetric.

The figure 3 presents the time-averaged flow structure around two surface-mounted buildings in the tandem array. Streamlines are plotted in vertical planes at four different values of $z / D$ of $0,0.2,0.4$ and 1 . The arrangement characterized by $H_{l} / H_{2}=0.6$ parameter, which is conducive to the occurrence of the so-called "downwash" effect [18]. This effect consists of washing the front side of the leeward object with large air masses, which results in strong air circulation in the area between cuboids, which determines flow structure between them. This situation is presented in Fig. 3a-c and Fig. 3e-g, which shows the result of numerical modeling (CFD) and experimental measurements (PIV) in the gap between the objects. The streamlines within this region, the structure of the recirculation, changes with height, indicating that the building wake has a three-dimensional structure. Fig. $3 \mathrm{~d}$ and $3 \mathrm{~h}$ present results for section located at $z / D=1$, where the flow tends to an undisturbed state by the presence of buildings arrangement. Similar conclusions result from Fig. 4, which presents the $\mathrm{x}$-component of mean velocity field $\left(U_{x} / U\right)$ and vectors magnitude around two surface-mounted buildings in the tandem array.

The results presented in this work show a good agreement between the PIV and the CFD methods. Note that some discrepancies are present near the ground, but as mentioned before, the PIV results are less reliable here. Fig. 4a and Fig. 4e show a very good agreement between the PIV and the CFD streamlines fields in the vertical axis plane. Both the PIV and CFD results show a standing vortex with similar size and position, in a gap between buildings and downstream of the leeward building.

The present study has focused on validation of CFD simulations of outdoor wind flow around configuration of simple system of buildings, and on the analysis of the flow phenomena at this particular scale in urban environments. The following limitations of the study are mentioned:

- The validation has only been performed for 3D steady RANS with the realizable kepsilon model for tandem configuration of buildings. Future studies should focus on the evaluation of different turbulence modelling approaches (RANS, DES and LES), different turbulence models, and also on different building configurations, including multi-zone buildings.

- The present study has only focused on flow parameters. Future work should focus on the analysis of physical and numerical simulation of pollutant dispersion in flow field in aspects of wind comfort in urban areas. 
CFD

a)

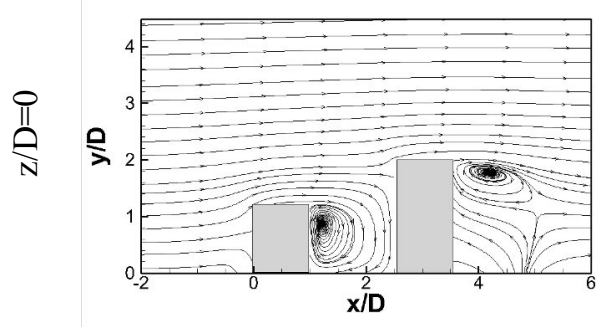

b)

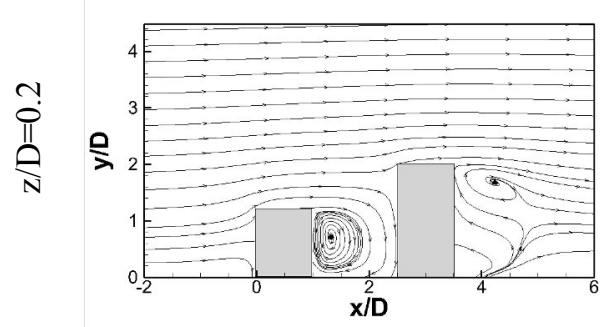

c)

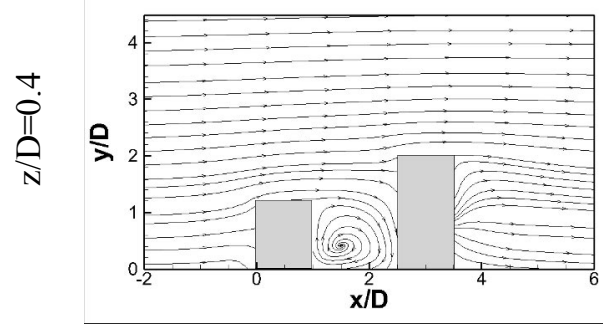

d)

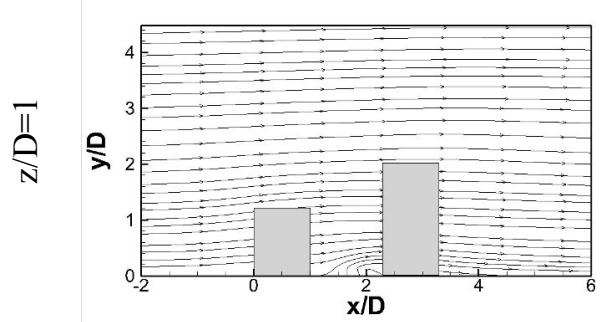

e)

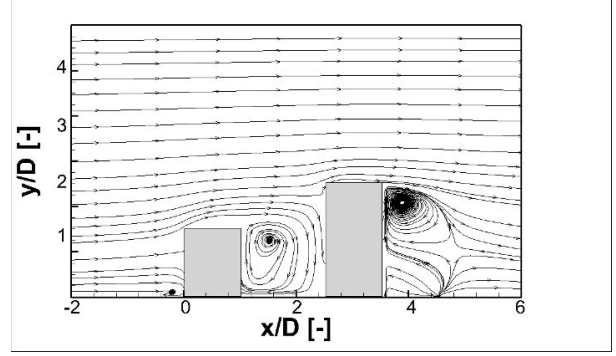

f)

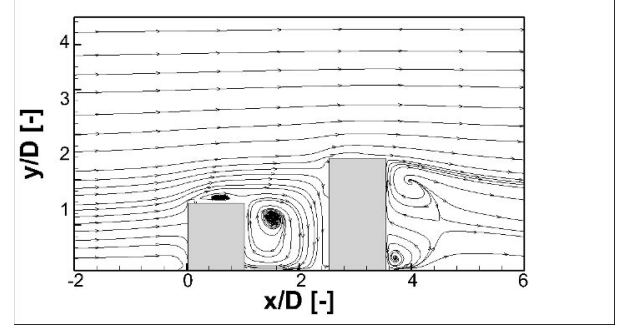

g)

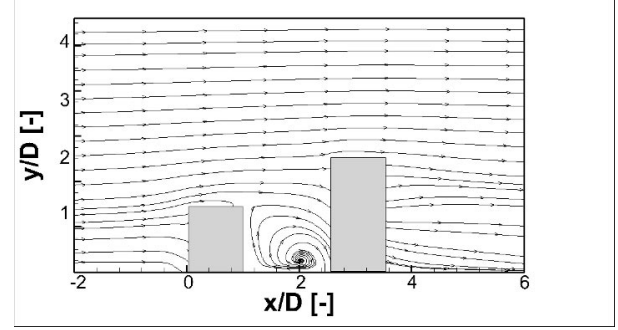

h)

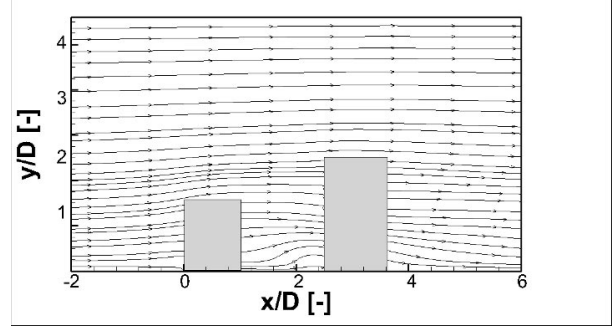

Fig. 3. Validation results: comparison of the numerical (CFD) results and the experimental (PIV) measurements in terms of streamlines in vertical planes at $z / D=0, z / D=0.2, z / D=0.4$ and $z / D=1$ around two surface-mounted buildings arranged in tandem. 
a)

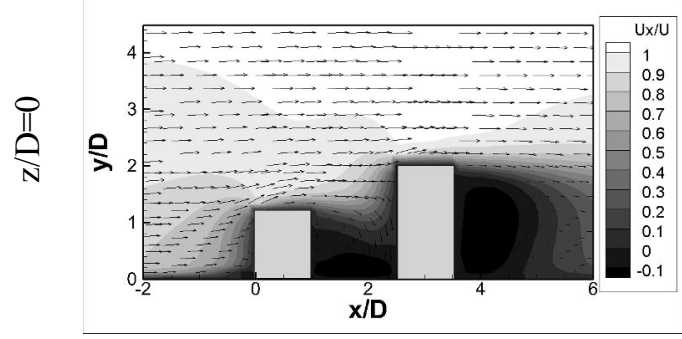

b)

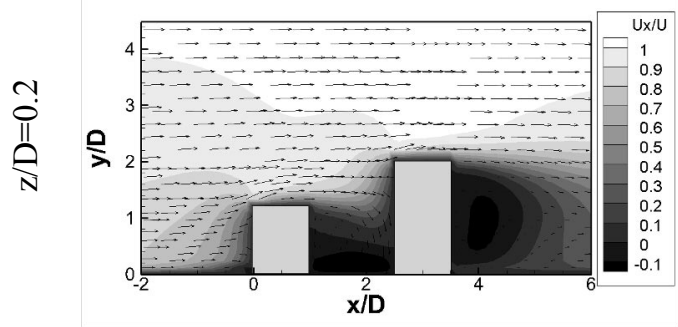

c)

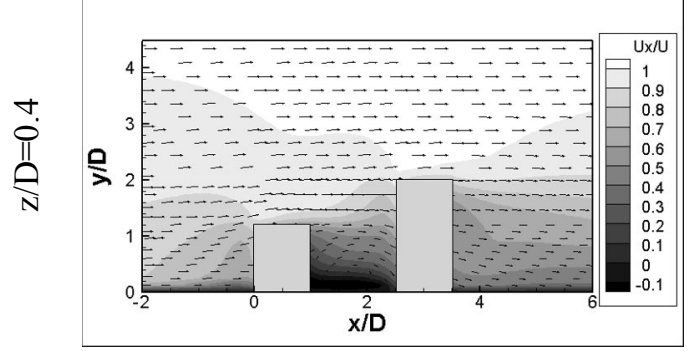

d)

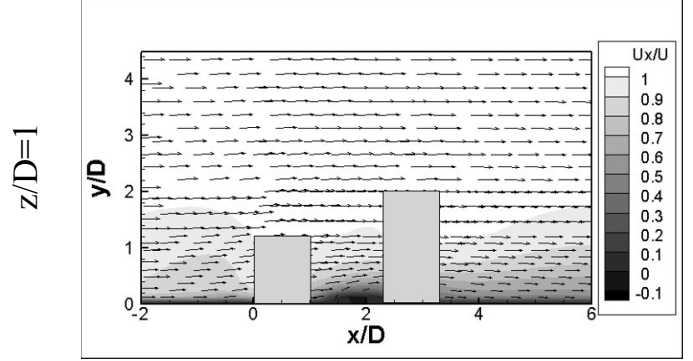

e)

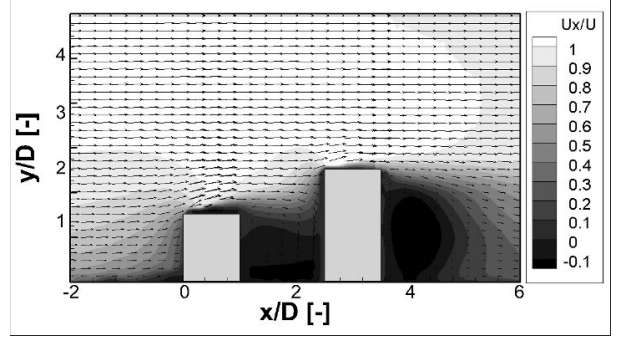

f)

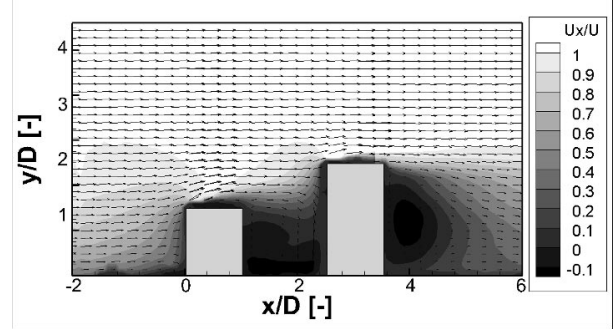

g)

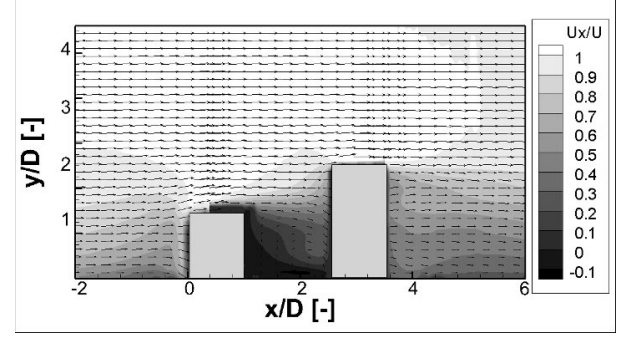

h)

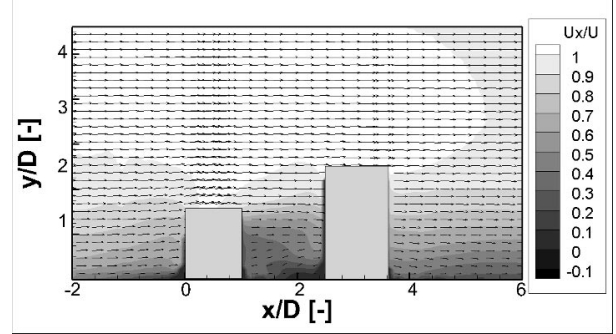

Fig. 4. Validation results: comparison of the numerical (CFD) results and the experimental (PIV) measurements in terms of x-component of mean velocity $U_{x} / U$ and vector magnitude velocity field in vertical planes at $z / D=0, z / D=0.2, z / D=0.4$ and $z / D=1$ around two surface-mounted buildings arranged in tandem. 
In spite of these limitations, it is important to mention that PIV experimental data will be beneficial to CFD modeling. This work will help to analyse the flow around the building in urban and suburban boundary layer and improve the understanding of the mechanism that influence the interference effects.

\section{Summary and conclusions}

Wind flow in built-up areas may be affected by a large range of parameters, mainly by wind velocity around buildings and adequate air quality. Those criteria are often in contradiction, because ensuring proper air quality requires adequate ventilation of built-up areas. Its effectiveness depends on wind direction, buildings configuration, etc. This paper has presented CFD simulations of wind-flow around buildings. The 3D steady ReynoldsAveraged Navier-Stokes (RANS) approach was applied with the realizable k-epsilon model. The intention of the paper was twofold: (1) to provide a validation study of outdoor wind flow around building arrangement by comparison with detailed Particle Image Velocimetry (PIV) measurements in an wind tunnel; (2) to provide information on the flow field and wind climate in urban areas. It is one of the important factors that must be taken into account in the process of architectural designing in urban organizations. Experimental and numerical analysis of the physical phenomena and mechanisms associated with airflow in the vicinity of objects in tandem arrangement immersed in a boundary layer made it possible to obtain a detailed description of the velocity field in the built-up environment.

The results of the validation study showed a good to very good agreement for analyzed configuration. The influence of the geometry on the fluid flow structure varies with height and is indicated mostly in the space between objects and also in the immediate vicinity of the ground. The application of both CFD numerical simulation of wind flow in a built environment with wind tunnel PIV experiment allowed to carry out verification and validation of results and also seems to be appropriate approach to proper planning of buildings configuration in order to achieve local wind comfort.

\section{References}

1. B. Blocken, J. Wind Eng. Ind. Aerodyn. 129 (2014)

2. B. Blocken, W.D. Janssen, T. van Hooff, Environ Model Softw. 30 (2012)

3. B. Blocken, T. Stathopoulos, J.P.A.J. van Beeck, Build Environ. 100 (2016)

4. Y. Hui, A. Yoshida, Y. Tamura, Y. Journal of Fluids and Structures, 37 (2013)

5. B. Blocken, Build Environ 91 (2015)

6. D.A. Paterson, C.J. Apelt, J. Wind Eng. Ind. Aerodyn. 35 (1990)

7. S. Murakami, A. Mochida, Y. Hayashi, S. Sakamoto, J. Wind Eng. Ind. Aerodyn. 44, 1-3 (1992)

8. T. Stathopoulos, J. Wind Eng. Ind. Aerodyn. 94, 11 (2006)

9. H. Sakamoto, H. Haniu, J. Wind Eng. and Ind. Aerodyn. 31, 41 (1988)

10. R.J. Martinuzzi, B. Havel, J. Fluid Eng. 122, 24 (2000)

11. T. Lawson, T.V. Lawson. Building aerodynamics. London: Imperial College Press, (2001)

12. T.H. Shih, W.W. Liou, A. Shabbir, Z. Yang, J. Zhu, Comput. Fluids. 24 (1995)

13. J. Franke, A. Hellsten, H. Schlünzen, B. Carissimo, Int J Environ Pollut. 44, 1-4 (2011)

14. Y. Tominaga, T. Okaze, A. Mochida, Build Environ. 46, 4 (2011)

15. P.J. Richards, R.P. Hoxey, J. Wind Eng. Ind. Aerodyn. 46\&47 (1993)

16. C.-H. Hu, F. Wang, Build Environ. 40 (2005)

17. B.E. Launder, D.B. Spalding, Comput Method Appl M. 3 (1974)

18. R. Gnatowska, Pol J Environ Stud. 24,4 (2015) 